\title{
On Infinitesimal Symmetries of the Self-Dual Yang-Mills Equations
}

\author{
T.A. IVANOVA \\ Bogoliubov Laboratory of Theoretical Physics, JINR, \\ 141980 Dubna, Moscow Region, Russia \\ E-mail: ita@thsun1.jinr.dubna.su \\ Received March 18, 1998; Accepted June 4, 1998
}

\begin{abstract}
Infinite-dimensional algebra of all infinitesimal transformations of solutions of the self-dual Yang-Mills equations is described. It contains as subalgebras the infinitedimensional algebras of hidden symmetries related to gauge and conformal transformations.
\end{abstract}

\section{Introduction}

Yang-Mills theory is a non-Abelian generalization of the Maxwell theory of electromagnetism. The dynamics of the non-Abelian gauge fields is described by the Yang-Mills (YM) equations, and the study of the space of solutions to the YM equations is of particular interest. In 1975 the equations giving a very important subclass of solutions to the YM equations were introduced [3]. These equations were called the self-dual Yang-Mills (SDYM) equations; their solutions provide absolute minima for the Yang-Mills functional in Euclidean 4-space. There exists a large literature on the geometric meaning of the SDYM equations (see e.g. $[1,12,18,11]$ ).

Our aim is to investigate infinitesimal symmetries of the SDYM equations. Under a symmetry we understand a transformation which maps solutions of the SDYM equations into solutions of these equations. In other words, symmetry transformations preserve the solution space. It is known that all local symmetries of the SDYM equations, which are also called manifest symmetries, are given by gauge transformations and conformal transformations. Since 1979, in a number of papers [14], it was shown that the SDYM equations have nonlocal, so-called 'hidden' symmetries which are related to global gauge transformations. More general gauge-type symmetries were described in $[16,15,5,9]$. In [13], an affine extension of conformal symmetries was introduced. The twistor interpretation of this algebra was discussed in [9]. But the problem of describing all possible (local and nonlocal) symmetries is not yet solved. 
The paper is organized as follows: In $\S \S 2,3$ we recall the main definitions (for more details see e.g. [6, 7]) and the Penrose-Ward twistor correspondence $[17,2,12,18,11]$. In $\S \S 4,5$ we give a cohomological description of the infinitesimal symmetries of the SDYM equations by reducing this problem to the problem of describing infinitesimal symmetries of holomorphic bundles over a twistor space.

\section{Definitions and notation}

2.1. The SDYM equations. Let us consider a principal fibre bundle $P=P\left(\mathbb{R}^{4}, S U(n)\right)$ over the Euclidean space $\mathbb{R}^{4}$ with the structure group $S U(n)$. Let $A_{\mu}(x)$ be components of the connection 1-form $A=A_{\mu}(x) d x^{\mu}$ in the bundle $P, x \in \mathbb{R}^{4}, \mu, \nu, \ldots=1, \ldots, 4$. Here and in what follows summation over repeated indices is understood. By definition, components $F_{\mu \nu}(x)$ of the curvature 2-form $F=\frac{1}{2} F_{\mu \nu}(x) d x^{\mu} \wedge d x^{\nu}$ in $P$ are $F_{\mu \nu}(x)=$ $\left[D_{\mu}, D_{\nu}\right]=\left[\partial_{\mu}+A_{\mu}, \partial_{\nu}+A_{\nu}\right]=\partial_{\mu} A_{\nu}-\partial_{\nu} A_{\mu}+\left[A_{\mu}, A_{\nu}\right], \partial_{\mu}:=\partial / \partial x^{\mu}$. Fields $A_{\mu}$ and $F_{\mu \nu}$ take values in the Lie algebra $s u(n)$.

The self-dual Yang-Mills (SDYM) equations have the form

$$
F_{\mu \nu}=\frac{1}{2} \varepsilon_{\mu \nu \rho \sigma} F_{\rho \sigma}
$$

where $\varepsilon_{\mu \nu \rho \sigma}$ is the completely antisymmetric tensor on $\mathbb{R}^{4}$ and $\varepsilon_{1234}=1$.

2.2. Manifest symmetries of the SDYM equations. As is known, eqs. (1) are invariant under the algebra of infinitesimal gauge transformations

$$
A_{\mu} \mapsto A_{\mu}^{\vartheta}=A_{\mu}+\delta_{\vartheta} A_{\mu}+\cdots, \quad \delta_{\vartheta} A_{\mu}=\partial_{\mu} \vartheta+\left[A_{\mu}, \vartheta\right], \quad \vartheta(x) \in \operatorname{su}(n)
$$

and under the algebra of infinitesimal conformal transformations

$$
A_{\mu} \mapsto A_{\mu}^{N}=A_{\mu}+\delta_{N} A_{\mu}+\ldots, \quad \delta_{N} A_{\mu}=N^{\nu} \partial_{\nu} A_{\mu}+A_{\nu} \partial_{\mu} N^{\nu},
$$

where a vector field $N=N^{\nu} \partial_{\nu}$ is any generator

$$
\begin{aligned}
& X_{a}=\delta_{a b} \eta_{\mu \nu}^{b} x_{\mu} \partial_{\nu}, \quad Y_{a}=\delta_{a b} \bar{\eta}_{\mu \nu}^{b} x_{\mu} \partial_{\nu}, \quad P_{\mu}=\partial_{\mu}, \\
& K_{\mu}=\frac{1}{2} x_{\sigma} x_{\sigma} \partial_{\mu}-x_{\mu} B, \quad B=x_{\sigma} \partial_{\sigma}, \quad a, b, \ldots=1,2,3,
\end{aligned}
$$

of the 15-parameter conformal group that is locally isomorphic to the group $S O(5,1)$ [7]. Here $\left\{X_{a}\right\}$ and $\left\{Y_{a}\right\}$ generate two commuting $S O(3)$ subgroups in $S O(4), P_{\mu}$ are the translation generators, $K_{\mu}$ are the generators of special conformal transformations and $B$ is the dilatation generator; $\eta_{\mu \nu}^{a}=\left\{\varepsilon_{b c}^{a}, \mu=b, \nu=c ; \delta_{\mu}^{a}, \nu=4 ;-\delta_{\nu}^{a}, \mu=4\right\}$ and $\bar{\eta}_{\mu \nu}^{a}=$ $\left\{\varepsilon_{b c}^{a}, \mu=b, \nu=c ;-\delta_{\mu}^{a}, \nu=4 ; \delta_{\nu}^{a}, \mu=4\right\}$ are the 't Hooft tensors satisfying

$$
\frac{1}{2} \varepsilon_{\mu \nu \rho \sigma} \eta_{\rho \sigma}^{a}=\eta_{\mu \nu}^{a}, \quad \frac{1}{2} \varepsilon_{\mu \nu \rho \sigma} \bar{\eta}_{\rho \sigma}^{a}=-\bar{\eta}_{\mu \nu}^{a}
$$

i.e. $\eta_{\mu \nu}^{a}$ are the self-dual tensors and $\bar{\eta}_{\mu \nu}^{a}$ are the anti-self-dual tensors.

2.3. Complex structure on $\mathbb{R}^{4}$. The most general constant complex structure $J=\left(J_{\mu}^{\nu}\right)$ on $\mathbb{R}^{4}$ has the form

$$
J_{\mu}^{\nu}=s_{a} \bar{\eta}_{\mu \sigma}^{a} \delta^{\sigma \nu} \Longrightarrow J_{\mu}^{\sigma} J_{\sigma}^{\nu}=-\delta_{\mu}^{\nu}
$$


where real numbers $s_{a}$ parametrize a two-sphere $S^{2}, s_{a} s_{a}=1, \bar{\eta}_{\mu \sigma}^{a}$ are the anti-self-dual 't Hooft tensor. Using $J$, one can introduce $(0,1)$ vector fields $\bar{V}_{\overline{1}}, \bar{V}_{\overline{2}}$

$$
\bar{V}_{\overline{1}}=\partial_{\bar{y}}-\lambda \partial_{z}, \quad \bar{V}_{\overline{2}}=\partial_{\bar{z}}+\lambda \partial_{y}
$$

satisfying $J_{\mu}^{\nu} \bar{V}_{\overline{1}, \overline{2}}^{\mu}=-i \bar{V}_{\overline{1}, \overline{2}}^{\nu}$. Here $y=x_{1}+i x_{2}, z=x_{3}-i x_{4}, \bar{y}=x_{1}-i x_{2}, \bar{z}=x_{3}+i x_{4}$ are the complex coordinates on $\mathbb{R}^{4} \simeq \mathbb{C}^{2}$, and $\lambda=\left(s_{1}+i s_{2}\right) /\left(1+s_{3}\right)$ is a local complex coordinate on $S^{2} \simeq \mathbb{C} P^{1}$.

2.4. Twistor space for $\mathbb{R}^{4}$. Let $C_{+}:=\left\{\lambda \in \mathbb{C P}^{1}:|\lambda| \leq 1+\alpha\right\}$, where $0<\alpha<1$ is a positive real number, $C_{-}:=\left\{\lambda \in \mathbb{C P}^{1}:|\lambda| \geq 1-\alpha\right\}$ (including $\lambda=\infty$ ). Then $C_{+}$and $C_{-}$form a two-set open cover of the Riemann sphere $\mathbb{C} P^{1}$ with the intersection $C_{\alpha}=C_{+} \cap C_{-}=\{\lambda: 1-\alpha \leq|\lambda| \leq 1+\alpha\}$. The vector field $\partial_{\bar{\lambda}}:=\partial / \partial_{\bar{\lambda}}$ is antiholomorphic $(0,1)$ vector field with respect to the standard complex structure $\varepsilon=i d \lambda \otimes \partial_{\lambda}-i d \bar{\lambda} \otimes \partial_{\bar{\lambda}}$ on $\mathbb{C} P^{1}$.

Twistor space $\mathcal{Z}$ of $\mathbb{R}^{4}$ is the bundle $\pi: \mathcal{Z} \rightarrow \mathbb{R}^{4}$ of complex structures on $\mathbb{R}^{4}$ associated with the principal $S O(4)$-bundle of orthogonal frames of $\mathbb{R}^{4}[1]$. It means that the fibre $\pi^{-1}(x)$ of $\mathcal{Z} \rightarrow \mathbb{R}^{4}$ over a point $x \in \mathbb{R}^{4}$ coincides with the space $\mathbb{C} P^{1}$ of complex structures on $\mathbb{R}^{4}$ defined above. The space $\mathcal{Z}$ is the trivial bundle over $\mathbb{R}^{4}$ with fibre $\mathbb{C} P^{1}$, hence $\mathcal{Z}=\mathbb{R}^{4} \times \mathbb{C} P^{1}$ is a manifold which can be covered by two coordinate patches:

$$
\mathcal{Z}=U_{+} \cup U_{-}, \quad U_{+}:=\left\{x \in \mathbb{R}^{4}, \lambda \in C_{+}\right\}, \quad U_{-}:=\left\{x \in \mathbb{R}^{4}, \lambda \in C_{-}\right\}
$$

with the intersection

$$
U:=U_{+} \cap U_{-}=\left\{x \in \mathbb{R}^{4}, \lambda \in C_{\alpha}=C_{+} \cap C_{-}\right\} .
$$

Let us denote the cover (6) by $\mathfrak{U}$.

The twistor space $\mathcal{Z}$ is a complex manifold with the complex structure $\mathcal{J}=(J, \varepsilon)$ on $\mathcal{Z}$. Vector fields $\bar{V}_{\overline{1}}, \bar{V}_{\overline{2}}$ from (5) and $\bar{V}_{\overline{3}}=\partial_{\bar{\lambda}}$ are the vector fields on $\mathcal{Z}$ of type $(0,1)$ with respect to the complex structure $\mathcal{J}$.

\section{The Penrose-Ward correspondence}

3.1. Complex vector bundle $\tilde{E}$ over the twistor space. Let $E=P \times_{S U(n)} \mathbb{C}^{n}$ be a complex vector bundle associated to $P$. Sections of this bundle are $\mathbb{C}^{n}$-valued functions depending on $x \in \mathbb{R}^{4}$.

By using the projection $\pi: \mathcal{Z} \rightarrow \mathbb{R}^{4}$, we can pull back the bundle $E$ with the connection $D=d x^{\mu} D_{\mu}$ to the bundle $\tilde{E}:=\pi^{*} E$ over $\mathcal{Z}=\mathbb{R}^{4} \times \mathbb{C} P^{1}$. By definition of the pull back, the pulled back connection $\tilde{D}:=\pi^{*} D$ will be flat along the fibres $\mathbb{C} P_{x}^{1}$ of the bundle $\mathcal{Z} \rightarrow \mathbb{R}^{4}$ and, therefore, the components of $\tilde{A}:=\pi^{*} A$ along the vector fields $\partial_{\lambda}, \partial_{\bar{\lambda}}$ in $\mathbb{C} P_{x}^{1}$ can be set equal to zero. Then we have $\tilde{D}=D+d \lambda \partial_{\lambda}+d \bar{\lambda} \partial_{\bar{\lambda}}$.

Local sections of the complex vector bundle $\tilde{E}$ are $\mathbb{C}^{n}$-valued functions defined on open subsets of $\mathcal{Z}=\mathbb{R}^{4} \times \mathbb{C P}^{1}$.

3.2. Linear system for the SDYM equations and holomorphic bundles. Let $\tilde{D}_{\bar{a}}^{(0,1)}(a=1,2,3)$ be components of $\tilde{D}$ along the $(0,1)$ vector fields $\bar{V}_{\bar{a}}$ on $\mathcal{Z}$. A section $\xi$ 
of the bundle $\tilde{E}$ is called a local holomorphic section if it is a local solution of the equations $\tilde{D}_{\bar{a}}^{(0,1)} \xi=0$ or, in local coordinates on $\mathcal{Z}$,

$$
\begin{aligned}
& \left(D_{\bar{y}}-\lambda D_{z}\right) \xi(x, \lambda, \bar{\lambda})=0, \\
& \left(D_{\bar{z}}+\lambda D_{y}\right) \xi(x, \lambda, \bar{\lambda})=0, \\
& \partial_{\bar{\lambda}} \xi(x, \lambda, \bar{\lambda})=0 .
\end{aligned}
$$

The equations $\tilde{D}_{\bar{a}}^{(0,1)} \xi=0$ on sections $\xi$ of the complex vector bundle $\tilde{E}$ define a holomorphic structure in $\tilde{E}$. Accordingly, the bundle $\tilde{E}$ is said to be holomorphic if eqs. (7), (8) are compatible, i.e. the $(0,2)$ components of the curvature of the bundle $\tilde{E}$ are equal to zero.

The solution of eq. (8) is $\xi(x, \lambda)$. Equations $(7)$ on $\xi(x, \lambda)$ are called the linear system for the SDYM equations $[4,17]$. It is easy to see that the compatibility conditions of the linear system (7) coincide with the SDYM equations written in the complex coordinates $y, z, \bar{y}, \bar{z}$ on $\mathbb{R}^{4} \simeq \mathbb{C}^{2}$.

Equations (7) have local solutions $\xi_{ \pm}(x, \lambda)$ over $U_{ \pm} \subset \mathcal{Z}$, and $\xi_{+}=\xi_{-}$on $U=U_{+} \cap U_{-}$ (for definitions of $U_{ \pm}$and $U$ see (6)). We can always represent $\xi_{ \pm}$in the form $\xi_{ \pm}=\psi_{ \pm} \chi_{ \pm}$, where $\psi_{ \pm}$are matrices of fundamental solutions of (7) defining a trivialization of $\tilde{E}$ over $U_{ \pm}$, and $\chi_{ \pm} \in \mathbb{C}^{n}$ are Čech fibre coordinates satisfying $\bar{V}_{\bar{a}} \chi_{ \pm}=0$ and $\chi_{-}=\mathcal{F} \chi_{+}$on $U=U_{+} \cap U_{-} \subset \mathcal{Z}$. The matrix $\mathcal{F}=\psi_{-}^{-1} \psi_{+}$is the transition matrix in the bundle $\tilde{E}$, i.e. holomorphic $S L(n, C)$-valued function on $U$ with non-vanishing determinant satisfying the conditions on transition matrices [8].

3.3. Ward's theorem. So, starting from the complex vector bundle $E$ over $\mathbb{R}^{4}$ with the self-dual connection $D$, we can construct the holomorphic vector bundle $\tilde{E}$ over $\mathcal{Z}$ with the transition matrix $\mathcal{F}=\psi_{-}^{-1} \psi_{+}$defined on $U \subset \mathcal{Z}$.

Conversely, if we are given the holomorphic vector bundle $\tilde{E}=\tilde{P}(\mathcal{Z}, S L(n, \mathbb{C})) \times_{S L(N, \mathbb{C})}$ $\mathbb{C}^{n}$ associated to the principal fibre bundle $\tilde{P}$ over $\mathcal{Z}$, which is holomorphically trivial on each fibre $\mathbb{C P}_{x}^{1}:\left.\tilde{E}\right|_{\mathbb{C} P_{x}^{1}} \simeq \mathbb{C} P_{x}^{1} \times \mathbb{C}^{n}$ (Ward's twistor construction [17]), then on $\mathbb{C} P_{x}^{1}$ the transition matrix $\mathcal{F}$ can be factorized in the form (Birkhoff's theorem):

$$
\mathcal{F}=\psi_{-}^{-1}(x, \lambda) \psi_{+}(x, \lambda),
$$

where $\psi_{ \pm}(x, \lambda)$ are $S L(n, \mathbb{C})$-valued functions holomorphic in $\lambda^{ \pm 1}$ on $C_{ \pm}$.

From the holomorphicity of $\mathcal{F}$ on $U\left(\bar{V}_{\bar{a}} \mathcal{F}=0\right)$ it follows that $\left(\bar{V}_{\bar{a}} \psi_{+}\right) \psi_{+}^{-1}=\left(\bar{V}_{\bar{a}} \psi_{-}\right) \psi_{-}^{-1}$ and, therefore,

$$
\begin{aligned}
& \left(\partial_{\bar{y}} \psi_{+}-\lambda \partial_{z} \psi_{+}\right) \psi_{+}^{-1}=\left(\partial_{\bar{y}} \psi_{-}-\lambda \partial_{z} \psi_{-}\right) \psi_{-}^{-1}=-\left(A_{\bar{y}}(x)-\lambda A_{z}(x)\right), \\
& \left(\partial_{\bar{z}} \psi_{+}+\lambda \partial_{y} \psi_{+}\right) \psi_{+}^{-1}=\left(\partial_{\bar{z}} \psi_{-}+\lambda \partial_{y} \psi_{-}\right) \psi_{-}^{-1}=-\left(A_{\bar{z}}(x)+\lambda A_{y}(x)\right),
\end{aligned}
$$

and the potentials $\left\{A_{\mu}\right\}$ defined by (10) satisfy the SDYM equations and do not change after transformations: $\psi_{ \pm} \mapsto \psi_{ \pm} h_{ \pm}$, where $h_{ \pm}$are regular holomorphic matrix-valued functions on $U_{ \pm}$. This means that the bundles with transition matrices $h_{-}^{-1} \mathcal{F} h_{+}$and $\mathcal{F}$ are holomorphically equivalent.

Let us summarize the facts about the Penrose-Ward correspondence in the theorem: 
Theorem $[\mathbf{2}, \mathbf{1}]$. There is a one-to-one correspondence between gauge equivalence classes of solutions to the SDYM equations in the Euclidean 4-space and equivalence classes of holomorphic vector bundles $\tilde{E}$ over the twistor space $\mathcal{Z}$, which are holomorphically trivial over each real projective line $\mathbb{C} P_{x}^{1}$ in $\mathcal{Z}$.

\section{Infinitesimal gauge-type symmetries}

4.1. The algebras $C^{0}(\mathfrak{U}, \mathcal{H})$ and $C^{1}(\mathfrak{U}, \mathcal{H})$. We consider the principal fibre bundle $\tilde{P}=$ $\tilde{P}(\mathcal{Z}, S L(N, \mathbb{C}))$ over the twistor space $\mathcal{Z}$ and the associated bundle $A d \tilde{P}=\tilde{P} \times_{\operatorname{AdSL}(n, \mathbb{C})}$ $\operatorname{sl}(n, \mathbb{C})$ with the adjoint action of the group $S L(n, \mathbb{C})$ on the algebra $\operatorname{sl}(n, \mathbb{C}): \xi \mapsto A d_{g} \xi=$ $g \xi g^{-1}, g \in S L(n, \mathbb{C}), \xi \in \operatorname{sl}(n, \mathbb{C})$. Let $\mathcal{H}$ be a sheaf of germs of holomorphic sections of the bundle $\operatorname{Ad} \tilde{P}$ (for definition see [8]), $\Gamma(\mathcal{U}, \mathcal{H})$ be a set of all sections of the sheaf $\mathcal{H}$ over an open $\operatorname{set} \mathcal{U} \subset \mathcal{Z}$.

A collection $\left\{\varphi_{+}, \varphi_{-}\right\}$of sections of $\mathcal{H}$ over the open sets $U_{+}$and $U_{-}$from (6a) is called a 0 -cochain over $\mathcal{Z}$, subordinate to the cover $\mathfrak{U}=\left\{U_{+}, U_{-}\right\}$. Thus, a 0 -cochain is an element of the space

$$
C^{0}(\mathfrak{U}, \mathcal{H}):=\Gamma\left(U_{+}, \mathcal{H}\right) \oplus \Gamma\left(U_{-}, \mathcal{H}\right)
$$

The space of 1 -cochains with values in $\mathcal{H}$

$$
C^{1}(\mathfrak{U}, \mathcal{H}):=\Gamma(U, \mathcal{H})
$$

is a set of sections $\varphi$ of the sheaf $\mathcal{H}$ over $U=U_{+} \cap U_{-}$. Notice that $C^{0}(\mathfrak{U}, \mathcal{H})$ and $C^{1}(\mathfrak{U}, \mathcal{H})$ are Lie algebras of holomorphic maps: $U_{ \pm} \rightarrow \operatorname{sl}(n, \mathbb{C})$ and $U \rightarrow \operatorname{sl}(n, \mathbb{C})$ respectively with pointwise commutator.

4.2. Action of $C^{1}(\mathfrak{U}, \mathcal{H})$ on transition matrices. The standard action of the algebra $C^{0}(\mathfrak{U}, \mathcal{H})$ on the space of holomorphic transition matrices $\mathcal{F}$ :

$$
\delta \mathcal{F}=\varphi_{-} \mathcal{F}-\mathcal{F} \varphi_{+}
$$

gives us holomorphically equivalent bundles. Hence, these transformations are trivial. But we shall consider the action of the algebra $C^{1}(\mathfrak{U}, \mathcal{H})$ on $\mathcal{F}$ :

$$
\delta_{\varphi} \mathcal{F}=\varphi(\lambda) \mathcal{F}+\mathcal{F} \varphi^{\dagger}\left(-\frac{1}{\bar{\lambda}}\right)
$$

where $\varphi \in C^{1}(\mathfrak{U}, \mathcal{H}), \varphi=\varphi(\lambda) \equiv \varphi(y-\lambda \bar{z}, z+\lambda \bar{y}, \lambda), \varphi(-1 / \bar{\lambda}) \equiv \varphi(y+\bar{z} / \bar{\lambda}, z-\bar{y} / \bar{\lambda},-1 / \bar{\lambda})$, and $\dagger$ denotes Hermitian conjugation.

Transformations (11) preserve the holomorphicity of $\mathcal{F}$ and preserve the hermiticity of the bundle $E$; they are local infinitesimal transformations of the transition matrix.

4.3. Infinitesimal gauge-type transformations of self-dual connections. Let us introduce the $\operatorname{sl}(n, \mathbb{C})$-valued smooth function $\phi$ on $U$ :

$$
\phi:=\psi_{-}\left(\delta_{\varphi} \mathcal{F}\right) \psi_{+}^{-1}=\psi_{-} \varphi(\lambda) \psi_{-}^{-1}+\psi_{+} \varphi^{\dagger}\left(-\frac{1}{\bar{\lambda}}\right) \psi_{+}^{-1}
$$


which is holomorphic in $\lambda \in C_{\alpha}$ and can be expanded in Laurent series

$$
\begin{aligned}
& \phi=\sum_{n=-\infty}^{\infty} \lambda^{n} \phi_{n}(x)=\phi_{-}-\phi_{+}, \\
& \phi_{+}:=\tilde{\phi}_{0}(x)-\sum_{n=1}^{\infty} \lambda^{n} \phi_{n}(x), \quad \phi_{-}:=\hat{\phi}_{0}(x)+\sum_{n=-\infty}^{-1} \lambda^{n} \phi_{n}(x), \quad \hat{\phi}_{0}(x)-\tilde{\phi}_{0}(x)=\phi_{0}(x) .
\end{aligned}
$$

The splitting $\phi=\phi_{-}-\phi_{+}$is a solution of the infinitesimal variant of the Riemann-Hilbert problem, and functions $\phi_{ \pm} \in \operatorname{sl}(n, \mathbb{C})$ are holomorphic in $\lambda \in C_{ \pm}$. It follows from (10) that $\tilde{D}_{\bar{a}}^{(0,1)} \phi=0$, and, therefore,

$$
\begin{aligned}
\left(D_{\bar{y}}-\lambda D_{z}\right) \phi_{+} & =\left(D_{\bar{y}}-\lambda D_{z}\right) \phi_{-}, \\
\left(D_{\bar{z}}+\lambda D_{y}\right) \phi_{+} & =\left(D_{\bar{z}}+\lambda D_{y}\right) \phi_{-} .
\end{aligned}
$$

The action of the algebra $C^{1}(\mathfrak{U}, \mathcal{H})$ on $S L(n, \mathbb{C})$-valued functions $\psi_{ \pm}$and on gauge potentials $\left\{A_{\mu}\right\}$ is given by the formulae

$$
\begin{aligned}
& \delta_{\varphi} \psi_{+}=-\phi_{+} \psi_{+}, \quad \delta_{\varphi} \psi_{-}=-\phi_{-} \psi_{-}, \\
& \delta_{\varphi} A_{\bar{y}}-\lambda \delta_{\varphi} A_{z}=D_{\bar{y}} \phi_{+}-\lambda D_{z} \phi_{+}=D_{\bar{y}} \phi_{-}-\lambda D_{z} \phi_{-}, \\
& \delta_{\varphi} A_{\bar{z}}+\lambda \delta_{\varphi} A_{y}=D_{\bar{z}} \phi_{+}+\lambda D_{y} \phi_{+}=D_{\bar{z}} \phi_{-}+\lambda D_{y} \phi_{-} .
\end{aligned}
$$

It follows from (14) that

$$
\begin{array}{ll}
\delta_{\varphi} A_{y}=\oint_{S^{1}} \frac{d \lambda}{2 \pi i \lambda^{2}}\left(D_{\bar{z}} \phi_{+}+\lambda D_{y} \phi_{+}\right), & \delta_{\varphi} A_{z}=-\oint_{S^{1}} \frac{d \lambda}{2 \pi i \lambda^{2}}\left(D_{\bar{y}} \phi_{+}-\lambda D_{z} \phi_{+}\right), \\
\delta_{\varphi} A_{\bar{y}}=\oint_{S^{1}} \frac{d \lambda}{2 \pi i \lambda}\left(D_{\bar{y}} \phi_{+}-\lambda D_{z} \phi_{+}\right), & \delta_{\varphi} A_{\bar{z}}=\oint_{S^{1}} \frac{d \lambda}{2 \pi i \lambda}\left(D_{\bar{z}} \phi_{+}+\lambda D_{y} \phi_{+}\right),
\end{array}
$$

where $S^{1}=\left\{\lambda \in C P^{1}:|\lambda|=1\right\}$. Thus, we have described the action of $C^{1}(\mathfrak{U}, \mathcal{H})$ on the space of solutions of SDYM equations.

Example 1. For $\varphi=0$ we have $\phi=0$. Choose $\phi_{+}=\phi_{-}=\vartheta(x), x \in \mathbb{R}^{4}$, then formulae (15) give us manifest gauge symmetries (2).

Example 2. If we choose $\varphi=\varphi(\lambda)$ (i.e. $\partial_{\mu} \varphi(x, \lambda)=0$ ), then obtain the action of the algebra $s u(n) \otimes C\left[\lambda, \lambda^{-1}\right]$ on the space of solutions of SDYM equations [14].

\section{$5 \quad$ Infinitesimal diffeomorphism-type symmetries}

5.1. The algebra $C^{0}(\mathfrak{U}, \mathcal{V})$. Let us consider a complexified tangent bundle $T^{\mathbb{C}}(\mathcal{Z})=$ $T^{(1,0)}(\mathcal{Z}) \oplus T^{(0,1)}(\mathcal{Z})$ of the twistor space $\mathcal{Z}$ and a sheaf $\mathcal{V}$ of germs of holomorphic sections of the bundle $T^{(1,0)}(\mathcal{Z})$. The set of all sections of the sheaf $\mathcal{V}$ over an open set $\mathcal{U} \subset \mathcal{Z}$ is denoted by $\Gamma(\mathcal{U}, \mathcal{V})$. If we take sections of $\mathcal{V}$ over each of the open sets $U_{+}$and $U_{-}$from the cover $\mathfrak{U}$, then the resulting collection of holomorphic vector fields is called a 0 -cochain over $\mathcal{Z}$, subordinate to the cover $\mathfrak{U}$. Thus, a 0 -cochain $\left\{\eta_{+}, \eta_{-}\right\}$is an element of the space

$$
C^{0}(\mathfrak{U}, \mathcal{V}):=\Gamma\left(U_{+}, \mathcal{V}\right) \oplus \Gamma\left(U_{-}, \mathcal{V}\right)
$$


The space of 1-cochains is defined as follows: $C^{1}(\mathfrak{U}, \mathcal{V}):=\Gamma(U, \mathcal{V})$, where $U=U_{+} \cap U_{-}$. Thus, elements of $C^{1}(\mathfrak{U}, \mathcal{V})$ are holomorphic vector fields $\eta_{+-}$defined on $U$.

5.2. Action of $C^{0}(\mathfrak{U}, \mathcal{V})$ on transition matrices. The vector space $C^{0}(\mathfrak{U}, \mathcal{V})$ can be described as the Lie algebra of holomorphic vector fields with pointwise commutator, defined on $U_{+}$and $U_{-}$. For any $\eta=\left\{\eta_{+}, \eta_{-}\right\} \in C^{0}(\mathfrak{U}, \mathcal{V})$ we define two actions of $C^{0}(\mathfrak{U}, \mathcal{V})$ on the transition matrix $\mathcal{F}$

$$
\delta_{\eta}^{ \pm} \mathcal{F}=\eta_{ \pm}(\mathcal{F})
$$

i.e. as a derivative of $\mathcal{F}$ along the vector fields $\eta_{ \pm} \in C^{0}(\mathfrak{U}, \mathcal{V})$.

One can also consider a combination of these actions:

$$
\delta_{\eta} \mathcal{F}=\delta_{\eta}^{-} \mathcal{F}-\delta_{\eta}^{+} \mathcal{F}
$$

It is easy to see that the algebra $C^{0}(\mathfrak{U}, \mathcal{V})$ acts on the algebra $C^{1}(\mathfrak{U}, \mathcal{H})$ by derivations, and we can consider a semidirect sum $C^{0}(\mathfrak{U}, \mathcal{V}) \dot{+} C^{1}(\mathfrak{U}, \mathcal{H})$ of these algebras.

5.3. Action of $C^{0}(\mathfrak{U}, \mathcal{V})$ on self-dual connections. Let us introduce the $\operatorname{sl}(n, C)$-valued smooth functions $\theta^{ \pm}$on $U$

$$
\theta^{ \pm}:=\psi_{-}\left(\delta_{\eta}^{ \pm} \mathcal{F}\right) \psi_{+}^{-1},
$$

which are holomorphic in $\lambda \in C_{\alpha}$ :

$$
\theta^{ \pm}=\sum_{n=-\infty}^{\infty} \lambda^{n} \theta_{n}^{ \pm}(x)=\theta_{-}^{ \pm}-\theta_{+}^{ \pm}
$$

where

$$
\theta_{+}^{ \pm}:=\tilde{\theta}_{0}^{ \pm}(x)-\sum_{n=1}^{\infty} \lambda^{n} \theta_{n}^{ \pm}(x), \quad \theta_{-}^{ \pm}:=\hat{\theta}_{0}^{ \pm}(x)+\sum_{n=-\infty}^{-1} \lambda^{n} \theta_{n}^{ \pm}(x), \quad \hat{\theta}_{0}^{ \pm}(x)-\tilde{\theta}_{0}^{ \pm}(x)=\theta_{0}^{ \pm}(x) .
$$

Thus, the functions $\theta_{ \pm}^{ \pm}(x, \lambda) \in \operatorname{sl}(n, C)$ are holomorphic in $\lambda^{ \pm 1} \in C_{ \pm} \subset \mathbb{C} P^{1}$.

For $\theta_{-}^{ \pm}$and $\theta_{+}^{ \pm}$we have

$$
\begin{aligned}
& \left(D_{\bar{y}}-\lambda D_{z}\right) \theta_{+}^{ \pm}=\left(D_{\bar{y}}-\lambda D_{z}\right) \theta_{-}^{ \pm}, \\
& \left(D_{\bar{z}}+\lambda D_{y}\right) \theta_{+}^{ \pm}=\left(D_{\bar{z}}+\lambda D_{y}\right) \theta_{-}^{ \pm} .
\end{aligned}
$$

The action of $C^{0}(\mathfrak{U}, \mathcal{V})$ on matrix-valued functions $\psi_{ \pm} \in S L(n, C)$ and on gauge potentials $\left\{A_{\mu}\right\}$ is given by the formulae

$$
\begin{aligned}
& \delta_{\eta}^{ \pm} \psi_{+}:=-\theta_{+}^{ \pm} \psi_{+}, \quad \delta_{\eta}^{ \pm} \psi_{-}:=-\theta_{-}^{ \pm} \psi_{-}, \\
& \delta_{\eta}^{ \pm} A_{\bar{y}}-\lambda \delta_{\eta}^{ \pm} A_{z}:=D_{\bar{y}} \theta_{+}^{ \pm}-\lambda D_{z} \theta_{+}^{ \pm}=D_{\bar{y}} \theta_{-}^{ \pm}-\lambda D_{z} \theta_{-}^{ \pm}, \\
& \delta_{\eta}^{ \pm} A_{\bar{z}}+\lambda \delta_{\eta}^{ \pm} A_{y}:=D_{\bar{z}} \theta_{+}^{ \pm}+\lambda D_{y} \theta_{+}^{ \pm}=D_{\bar{z}} \theta_{-}^{ \pm}+\lambda D_{y} \theta_{-}^{ \pm} .
\end{aligned}
$$


It follows from (19) that

$$
\begin{array}{ll}
\delta_{\eta}^{ \pm} A_{y}=\oint_{S^{1}} \frac{d \lambda}{2 \pi i \lambda^{2}}\left(D_{\bar{z}} \theta_{+}^{ \pm}+\lambda D_{y} \theta_{+}^{ \pm}\right), & \delta_{\eta}^{ \pm} A_{z}=-\oint_{S^{1}} \frac{d \lambda}{2 \pi i \lambda^{2}}\left(D_{\bar{y}} \theta_{+}^{ \pm}-\lambda D_{z} \theta_{+}^{ \pm}\right), \\
\delta_{\eta}^{ \pm} A_{\bar{y}}=\oint_{S^{1}} \frac{d \lambda}{2 \pi i \lambda}\left(D_{\bar{y}} \theta_{+}^{ \pm}-\lambda D_{z} \theta_{+}^{ \pm}\right), & \delta_{\eta}^{ \pm} A_{\bar{z}}=\oint_{S^{1}} \frac{d \lambda}{2 \pi i \lambda}\left(D_{\bar{z}} \theta_{+}^{ \pm}+\lambda D_{y} \theta_{+}^{ \pm}\right),
\end{array}
$$

where $S^{1}=\left\{\lambda \in \mathbb{C} P^{1}:|\lambda|=1\right\}$.

Example 3. Let us consider the holomorphic vector fields $\eta=\lambda^{-n} \tilde{N}$ on $U \subset \mathcal{Z}, n=$ $0, \pm 1, \pm 2, \ldots$, where $\tilde{N}$ are vector fields on $\mathcal{Z}$ realizing the action of $s o(5,1)$ on $\mathcal{Z}$, which preserves the holomorphicity of the bundle $\tilde{E} \rightarrow \mathcal{Z}$. Such lift $N \rightarrow \tilde{N}$ of vector fields from $\mathbb{R}^{4}$ to $\mathcal{Z}$ was described in [10]. As it has been shown in [9], symmetries (20) for $\eta=\lambda^{-n} \tilde{N}$ with $n \geq 0$ are in one-to-one correspondence with the symmetries from [13].

\section{Conclusion}

To sum up, using the one-to-one correspondence between the classes of holomorphically equivalent transition matrices $\mathcal{F}$ and the gauge equivalent classes of self-dual connections, to any infinitesimal transformations (11) and (16) of transition matrices we have associated the infinitesimal transformations (15) and (20) of solutions $\left\{A_{\mu}\right\}$ of the SDYM equations. There are no other infinitesimal automorphisms of the bundle $\tilde{E}$ over $\mathcal{Z}$ besides those generated by the algebras $C^{0}(\mathfrak{U}, \mathcal{V})$ and $C^{1}(\mathfrak{U}, \mathcal{H})$. Thus, the infinite-dimensional algebra of all infinitesimal transformations of solutions of the SDYM equations has the form $C^{0}(\mathfrak{U}, \mathcal{V}) \dot{+} C^{1}(\mathfrak{U}, \mathcal{H})$.

Notice that all the results of this paper may be generalized to the case of the SDYM equations in $4 n$-dimensional spaces considered e.g. in [19]. It would be interesting to generalize our results to other SDYM-type equations in dimension greater than four (see e.g. [20]), various modifications of which arise in string and membrane theories (see e.g. [21] and references therein).

\section{Acknowledgements}

The author is grateful to Ina Kersten, Sylvie Paycha and Sheung Tsun Tsou for encouraging and helpful discussions. This work is supported in part by the grant No. 98-01-00173.

\section{References}

[1] Atiyah M.F., Hitchin N.J. and Singer I.M., Proc. R. Soc. Lond. A, 1978, V.362, 425-461.

[2] Atiyah M.F. and Ward R.S., Commun. Math. Phys., 1977, V.55, 117-124.

[3] Belavin A.A., Polyakov A.M., Schwarz A.S. and Tyupkin Yu.S., Phys. Lett. B, 1975, V.59, 85-87.

[4] Belavin A.A. and Zakharov V.E., Phys. Lett. B, 1978, V.73, 53-57.

[5] Crane L., Commun. Math. Phys., 1987, V.110, 391-414.

[6] Daniel M. and Viallet C.M., Rev. Mod. Phys., 1980, V.52, 175-196. 
[7] Dubrovin B.A., Fomenko A.T. and Novikov S.P., Modern Geometry, Springer, Berlin, 1984.

[8] Griffits P. and Harris J., Principles of Algebraic Geometry, John Wiley \& Sons, New York, 1978.

[9] Ivanova T.A., J. Math. Phys., 1998, V.39, 79-87.

[10] Legaré M. and Popov A.D., Phys. Lett. A, 1995, V.198, 195-200.

[11] Mason L.J. and Woodhouse N.M.J., Integrability, Self-Duality and Twistor Theory, Clarendon Press, Oxford, 1996.

[12] Penrose R. and Rindler W., Spinors and Space-Time, Vol.2, Cambridge University Press, Cambridge, 1986.

[13] Popov A.D. and Preitschopf C.R., Phys. Lett. B, 1996, V.374, 71-79.

[14] Prasad M.K., Sinha A. and Chau Wang L.-L., Phys. Lett. B, 1979, V.87, 237-238; Pohlmeyer K., Commun. Math. Phys., 1980, V.72, 37-47;

Chau L.-L., Ge M.-L. and Wu Y.-S., Phys. Rev. D, 1982, V.25, 1086-1094;

Chau L.-L., Ge M.-L., Sinha A. and Wu Y.-S., Phys. Lett. B, 1983, V.121, 391-396;

Chau L.-L., Lect. Notes Phys., 1983, V.189, 111-127;

Dolan L., Phys. Lett. B., 1982, V.113, 387-390; Phys. Rep., 1984, V.109, 1-94;

Avan J. and de Vega H.J., Int. J. Mod. Phys. A, 1988, V.3, 1263-1299.

[15] Takasaki K., Commun. Math. Phys., 1984, V.94, 35-59.

[16] Ueno K. and Nakamura Y., Phys. Lett. B, 1982, V.109, 273-278.

[17] Ward R.S., Phys. Lett. A., 1977, V.61, 81-82.

[18] Ward R.S. and Wells R.O. Jr., Twistor Geometry and Field Theory, Cambridge University Press, Cambridge, 1990.

[19] Ward R.S., Nucl. Phys. B, 1984, V.236, 381-396; Galperin A., Ivanov E., Ogievetsky V. and Sokatchev E., Ann. Phys., 1988, V.185, 1-21; Ivanova T.A. and Popov A.D., Theor. Math. Phys., 1993, V.94, 225-242.

[20] Corrigan E., Devchand C., Fairlie D.B. and Nuyts J., Nucl. Phys. B, 1983, V.214, 452-468;

Fairlie D.B. and Nuyts J., J. Phys. A, 1984, V.17, 2867-2872;

Fubini S. and Nicolai H., Phys. Lett. B., 1985, V.155, 369-372;

Ivanova T.A. and Popov A.D., Lett. Math. Phys., 1992, V.24, 85-92;

Ivanova T.A. and Popov A.D., J. Math. Phys., 1993, V.34, 674-680;

Baulieu L., Kanno H. and Singer I.M., hep-th/9704167;

Hull C.M., hep-th/9710165;

Figueroa-O'Farrill J.M., hep-th/9710168;

Abdel-Khalek K., hep-th/9710177.

[21] Harvey J.A. and Strominger A., Phys. Rev. Lett., 1991, V.66, 549-552;

Ivanova T.A., Phys. Lett. B., 1993, V.315, 277-282;

Günaydin M. and Nicolai H., Phys. Lett. B., 1995, V.351, 169-172;

Curtright T., Fairlie D. and Zachos C., Phys. Lett. B, 1997, V.405, 37-44;

Floratos E.G. and Leontaris G.K., hep-th/9710064, hep-th/9802018;

Castro C. and Plebanski J., hep-th/9710041;

Floratos E.G., Leontaris G.K., Polychronakos A.P. and Tzani R., hep-th/9711044. 